\title{
NUMERICAL SIMULATIONS OF OPTICAL KNOTS IN YSO OUTFLOWS
}

\author{
F. RUBINI AND S. LORUSSO \\ Dip. Astronomia, Univ. Florence \\ Largo E. Fermi 5, 50125 Florence, Italy \\ C. GIOVANARDI \\ Osservatorio Astrofisico di Arcetri \\ Largo E. Fermi 5, 50125 Florence, Italy \\ AND

\section{F. LEEUWIN} \\ Dip. Fisica, Univ. Bologna \\ Viale Berti-Pichat 2-6, 40126 Bologna, Italy
}

\begin{abstract}
The aim of this work is to show that, though 3D effects should be invoked to explain the less regular arc-like regions, the internal knotty emissions may be explained through a roughly axisymmetric pattern of oblique shocks.

To this purpose, numerical simulations of non-equilibrium, radiative, hydrodynamical jets in axisymmetric geometry have been performed. After showing that the internal reflecting waves, travelling in the beam of a stellar jet, steepen into oblique shocks, we verify that the optical emission arising from the shocked regions is in good agreement with observations.

Since the optical emission strongly depends on the physical and chemical parameters, (namely temperature and electron densities inside the emitting regions), special care is devoted to solving the energy transport equation.
\end{abstract}

\section{Introduction}

The most recent observations of $\mathrm{HH}$ objects show that the emission regions are, in some cases, far from being simple axially aligned structures of equally spaced knots. Instead, they reveal a morphological variety with a wide range of shapes and spatial scales. 
Very recent images of HH47 from HST, for instance, reveal interesting details of a quite complex structure, where the emission in [SII] seems to be decoupled from that in $\mathrm{H} \alpha$. The former fills the whole body of the jet and, in its inner and faster part, forms a sinuous chain of small scale knots, the spacing being of 2" or 3", the latter is concentrated in wisps and filaments. The difference $\mathrm{H} \alpha$ - [SII] image clearly shows that the knots in [SII] are internal to the flow whereas the filaments in $\mathrm{H} \alpha$ are localized at the surface of the beam, (Heathcote et al., 1996). These authors argue that it is just the interaction between the beam and the neutral external medium that allows the formation of the observed thin $\mathrm{H} \alpha$ Balmer-dominated filaments, where sudden changes in the direction of the flow drive the formation of secondary bow-shocks. At the same time, the source of HH47 seems to undergo temporal variations at intervals of $500-1000$ years, creating multiple leading bow-shocks whose spacing is much larger than the one between knots, a behavior typical of many $\mathrm{HH}$ objects.

These observations suggest that more than one mechanism is at work to produce the optical emission, which, with respect to the spacing between two separate emissions, could be classified as large scale and small scale processes, respectively.

The arc-like $\mathrm{H} \alpha$ dominated regions, associated with the interaction between the flow and medium, extend over both large and small scales, resp. in the form of leading, and secondary, bow-shocks. The former would directly depend on the temporal variation of the outbursts, the latter on strong deviations of the trajectories of the particles, maybe due to the growth of kink-like instabilities, to precession of the source of the jet, or interaction with a non-homogeneous external medium. These effects could of course act separately, or together.

On the other hand, the internal, knotty emission, like the ones that are well observed in HH34, HH211, as well as in HH47, seem to be essentially small scale events, which could arise from high-frequency ( $\Delta t$ of a few years) perturbations of the inflow conditions rapidly steepening into shocks (Stone et al., 1993, Suttner et al., 1997), or from pinch-like instabilities originating from the toroidal component of the external magnetic field (Hardee et al., 1997).

In this work we show that it is not necessary to invoke rapid fluctuations of the inflow conditions to explain the small scale structures inside the beam of the jet. Even neglecting the presumably important magnetic effects, a pattern of emission is produced, within a purely hydrodynamic model, by internal oblique shocks.

To this purpose we have performed numerical simulations of radiative jets, fed by a stationary source, propagating into the cold, undisturbed ISM.

A recent diagnostic model allows to deduce, for the emitting regions, 
average values for the gas temperature, total density and ionization fraction (Bacciotti et al., 1995). In practice, we span a range of possible inflow values searching for those that lead to the right parameters for the emitting knots.

Our simulations show that internal reflecting waves can survive energy losses and steepen into oblique shocks. The compressed gas in post-shock regions is the source of optical emission, whose knotty pattern arises from the shock pattern. Emissivity in [SII] and $\mathrm{H} \alpha$, with the proper choice of the inflow parameters, has been found to be in good agreement with observations.

Special care has been taken in treating energy losses and the cooling function. The diagnostic model of Bacciotti shows that the emitting gas average temperature is $\approx 7000 \mathrm{~K}$, which means that the cooling function should also take into account temperatures below $10^{4} \mathrm{~K}$, a lower bound in many numerical simulations in the literature.

\section{Non-stationary dynamics of stellar jets and formation of in- ternal shocks}

Stellar jets are non-stationary and non-periodic hypersonic flows whose internal structure evolves in space and time depending on the interaction between the internal beam, the surrounding region filled with the jet particles that, at the head, have been slowed and deviated by the Mach disk, the so-called cocoon, and the shroud, the external matter entrained by the bow shock. The pressure gradient between these structures drives the formation of internal oblique shocks, and it is this mechanism which we intend to investigate as the agent responsible for optical knots.

In under-expanded jets, such as those we consider, the jet particles which leave the nozzle undergo a sudden, quasi-adiabatic expansion, which makes the trajectories diverge and the pressure decrease. Once the equilibrium pressure with the cocoon is reached, compressional waves start to travel towards the axis, and eventually steepen to form the first oblique shock, causing particles to converge into the first compression region. An expansion phase follows, after which the mechanism repeats generating a knotty structure. At its end, the particles are slowed by the Mach disk, before being deviated into the cocoon.

At the same time, particles from the external medium which have been entrained and warmed by the bow-shock, cool, forming a thick layer of fast external matter right behind the bow-shock itself. As a consequence, a shroud forms between the bow-shock and the cocoon, where, due to the low density and the large cooling length, local pressure keeps high enough to embed the jet cocoon. The relative importance of the cocoon and the shroud may be roughly measured by the relative shock number, which is 
the ratio between the cooling lengths of the bow-shock and the Mach disk:

$$
\frac{\xi_{b s}}{\xi_{M d}} \approx \eta^{3}
$$

(Blondin et al., 1990), where $\eta \equiv \rho_{\text {jet }} / \rho_{I S M}$ is the density ratio. This means that light jets behave as if they were adiabatic jets, with extended highly embedding cocoons flowing into an isothermal medium, whereas the shroud is dominant in heavy jets.

It follows that we should investigate jets where the inflow pressure ratio $P_{r} \equiv \frac{P_{j e t}}{P_{I S M}}$ is as low as possible, as they should be rapidly embedded by the cocoon and develop internal shocks. In the following we shall see whether this choice is consistent with the constraints arising from observations.

As for the inflow radius $r_{j e t}$, it is well known that the spacing between oblique shocks increases with radius. Therefore we adopt for $r_{j e t}$ a value significantly smaller than the value usually assumed for stellar outflows in numerical simulations (but consistent with the hypothesis that flows emanate from the central zone of circumstellar disks). We recall however that in under-expanded jets the effective jet radius, i.e. the radius of the pinching channel bounding the supersonic flow, is larger than the inflow nozzle radius, $r_{j e t}$. Namely, it increases with the pressure ratio $P_{r}$, tending to $r_{j e t}$ only in the limit $P_{r}=1$.

As a conclusion, keeping the values of both the pressure ratio $\frac{P_{\text {jet }}}{P_{I S M}}$ and the inflow radius $r_{j e t}$ as small as possible (within observational constraints) should lead to the expected pattern of small scale optical knots.

\section{Jet parameters and observational constraints}

Though, for given external medium parameters, internal oblique shocks exist for a wide range of the inflow parameters $\left(r_{j e t}, P_{j e t}\right)$, the set leading to correct values of temperature and density in the emitting regions is a small subdomain.

The already cited diagnostic technique (Bacciotti et al., 1995) allows one to derive average values for the emitting gas temperature, total density and ionization fraction in the optical knots, starting from the observed values of emissivity ratios, namely, $[O I] /[N I I],[N I I] / H \alpha,[S I I] / H \alpha$. For HH34 the mean temperature in the knots is estimated to range from 5000 to $7000 \mathrm{~K}$, while the electron density ranges from 100 to $1000 \mathrm{~cm}^{-3}$ and the ionization fraction $x$ decreases from 0.2 to 0.032 along the flow, corresponding to a total density of about $5000-20000 \mathrm{~cm}^{-3}$ (Bacciotti \& Eislöffel, 1997).

Though it would be difficult to use directly these results to infer the inflow parameters in our simulations, we can use them as a $a$ posteriori check. 
The total density at the inflow, $\rho_{j e t}$, is assumed to be equal to the density in knots. The same value is taken for the undisturbed ISM $(\eta=1)$; its initial temperature is of order $100 \mathrm{~K}$ (with the corresponding pressure deduced from a perfect gas equation). This necessarily leads to under-expanded jets, with pressure ratios $P_{r}$ greater than one; an inflow material which is too cold $\left(P_{r} \sim 1\right)$ has no chance of reaching the correct emitting temperature, not even through compression by oblique shocks.

In fact, while the assumption of low values for $P_{j e t}$, as already pointed out, favours the formation of oblique shocks, a lower limit for $P_{j e t}$ arises from the requirement that significant emissions in [SII] and $\mathrm{H} \alpha$ be produced, since this implies temperatures of order $7000 \mathrm{~K}$.

Two regimes of pressure have been explored numerically:

- Moderately under-expanded jets:

case A) $\rho_{\text {jet }}=5000 \mathrm{~cm}^{-3}, x_{j e t}=0.3, T_{\text {jet }} \approx 3000 \mathrm{~K}, P_{r}=30$;

- Strongly under-expanded jets:

case B) $\rho_{\text {jet }}=5000 \mathrm{~cm}^{-3}, x_{j e t}=0.3, T_{j e t} \approx 6000 \mathrm{~K}, P_{r}=60$;

The simulations show that only when the inflow temperature $T_{j e t}$ is at least $\approx 5000 \mathrm{~K}$ does the post-shock temperature $T_{k n o t s}$ reach the value of $7000 \mathrm{~K}$, and the emissivity in $[S I I]$ and $\mathrm{H} \alpha$ appears in agreement with the observed data.

Regarding the inflow radius, simulations with either $r_{j e t}=0.2 \cdot 10^{15} \mathrm{~cm}$ and $r_{j e t}=10^{15} \mathrm{~cm}$ have been performed.

\section{The model and the numerical code}

\subsection{CHEMICAL EVOLUTION AND RELATED ENERGY TERMS}

We consider a gas composed of molecular, atomic and ionized hydrogen plus helium and solar abundancy metals, and we describe this system via the Euler equations modified to take into account radiative losses, plus two equations that describe the non-equilibrium evolution of the electron population and of molecular hydrogen.

total density conservation + total momentum conservation + total energy conservation $-S_{\text {rad }}+S_{i o n}+S_{H 2}$ (energy source) + $\frac{\partial N_{e-}}{\partial t}+\nabla \cdot\left(N_{e^{-}} \mathbf{u}\right)=N_{e s}+$ $\frac{\partial H_{2}}{\partial t}+\nabla \cdot\left(H_{2} \mathbf{u}\right)=N_{h s}$

The source term in the electron equation is given by: $N_{e s}=N_{i o n}-N_{r e c}$, where the recombination rate $N_{\text {rec }}$ is taken from Spitzer (1978) and the ionization rate $N_{i o n}$ is given by Lang (1975). This yields the contribution $S_{i o n}=13.6 \mathrm{eV} \cdot N_{e s}$ to the total energy variation. 
The source term in the molecular hydrogen equation, $N_{h s}$, arises only from molecular dissociation, due to collisions with $\mathrm{H}_{2}$ and HI (Lepp \& Shull 1983), and HII and electrons (Hollenbach \& McKee, 1989, Mac Low $\&$ Shull, 1986, Martin et al., 1996). The corresponding contribution to the total energy source is $S_{H 2}=\Lambda_{\text {rad }}+\Lambda_{\text {diss }}$, where:

$-\Lambda_{\text {diss }}=N_{h s} \cdot 4.48 \mathrm{eV}$ results from dissociations

$-\Lambda_{\text {rad }}$ is due to collisions with $\mathrm{HI}$ and $\mathrm{H}_{2}$ and is taken from Lepp \& Shull (1983).

The cooling function takes into account temperatures also below $10^{4} \mathrm{~K}$. Namely, for $T>10^{4} \mathrm{~K}$ the usual cooling function by Dalgarno \& Mc Cray (1972) has been used, which considers radiative losses due to de-excitation of $\mathrm{HI}$ energy levels.

For $T<10^{4} \mathrm{~K}$, the cooling function is extended by computing energy losses mainly due to collisions between electrons and $\mathrm{C}^{+}, \mathrm{O}, \mathrm{N}, \mathrm{Fe}^{+}, \mathrm{O}^{+}, \mathrm{S}^{+}$, or, for still lower temperatures, between $\mathrm{HI}$ and $\mathrm{C}^{+}, \mathrm{O}, \mathrm{Si}^{+}, \mathrm{Fe}^{+},($Bacciotti et al., 1995).

\subsection{NUMERICAL CODE}

The numerical scheme is based on a second order HLLE Riemann solver, (Einfeldt, 1991) for the spatial derivatives, combined with a second order multistage TVD-Runge Kutta time integration (Shu, 1988).

A mesh with variable spacing along the $z$ direction was used, with at least $600 \times 100$ points, at most $2500 \times 100$ points, in $R, z$.

\section{Numerical results}

The simulations we report on differ essentially through the pressure ratio, and eventually the inflow radius. All runs assume a density ratio $\eta=1$.

A moderately under-expanded case, with inflow parameters:

$\rho_{\text {jet }}=5000 \mathrm{~cm}^{-3}, T_{\text {jet }}=3000 \mathrm{~K}$, and $r_{j e t}=10^{15} \mathrm{~cm}$, and with pressure ratio $P_{r}=30$ (corresponding to $T_{I S M}=100 \mathrm{~K}$ ), showed no significant emission. Though a pattern of compression regions formed in the channel of the flow, the temperature in the knots remained indeed too low $(1500 K)$.

Fig. 1 to 3 show instead a strongly under-expanded jet $\sim 1300$ years old. 


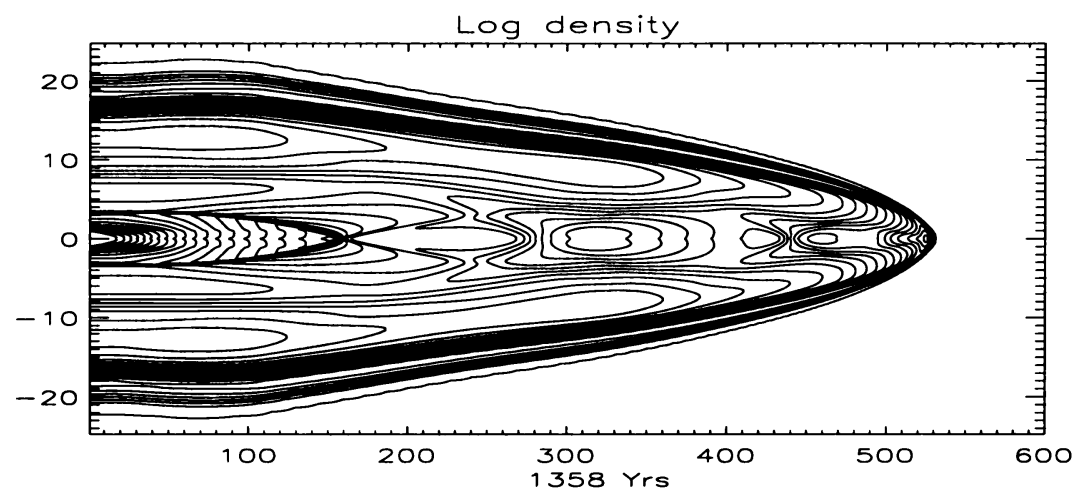

Figure 1. Density map (logarithmical) for the strongly under-expanded case with nozzle radius $r_{j e t}=10^{15} \mathrm{~cm}$

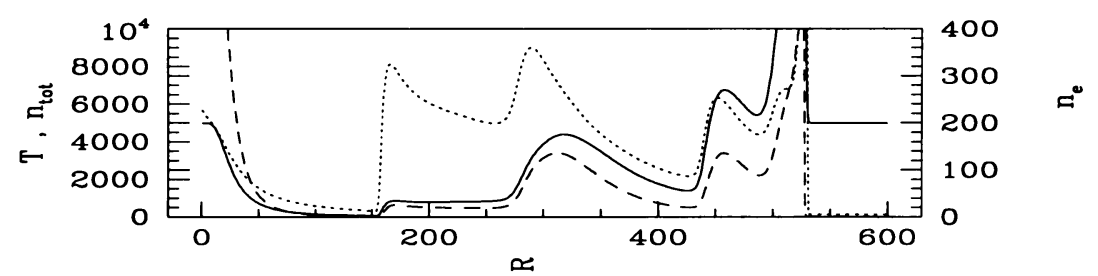

Figure 2. Profile along the jet axis of temperature (dotted line), electrons density (in $\mathrm{cm}^{-3}$, dashed line) and total density (in $\mathrm{cm}^{-3}$, full line).

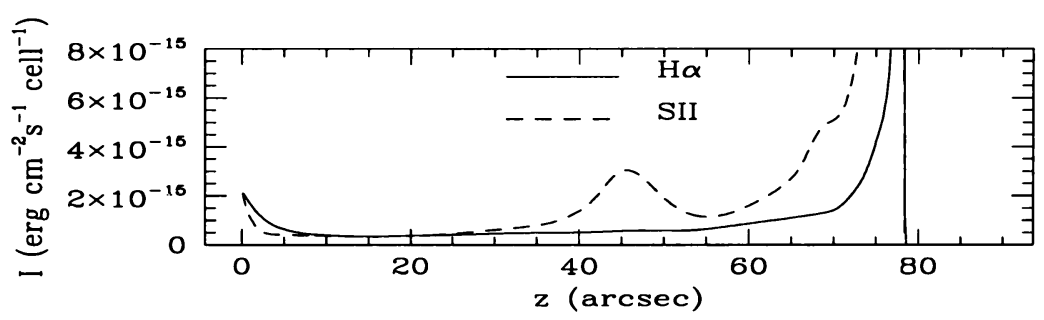

Figure 3. Profile along the jet axis of the [SII] (dashed line) and $\mathrm{H}_{\alpha}$ (full line) fluxes, at a distance of $460 \mathrm{pc}$, from a cylindrically symmetric slice normal to the axis, with a width of 1 cell $\left(1.2 \times 10^{14} \mathrm{~cm}\right)$. 
The external parameters are the same as before, whereas the jet pressure now corresponds to a pressure ratio $P_{r}=60\left(T_{j e t} \approx 6000 \mathrm{~K}\right)$. The radius is again $r_{j e t}=10^{15} \mathrm{~cm}$. In the density map displayed in Fig. 1, the expansion phase extends over $\approx 1.5 \cdot 10^{17} \mathrm{~cm}$, and the first knot appears at a distance from the source of $\approx 3 \cdot 10^{17} \mathrm{~cm}$. The expansion, as well as the knots, are clearly traced by the temperature and density profiles of Fig. 2 . The spacing between the first and the second knot is of order $2 \cdot 10^{17} \mathrm{~cm}$, too large with respect to the expected values. However, the emissivity results within the good range. Fig. 3 shows the emissivity in [SII] and $\mathrm{H} \alpha$, integrated over the beam, versus the axis (the length units are given in arcsec, having assumed a distance of $460 \mathrm{pc}$ ). The units for the vertical axis are erg $\mathrm{cm}^{-2} \mathrm{~s}^{-1}$ per numerical cell, thus we need to add the contributions of all cells inside the knot to compare with observations. Since we have $\approx 50$ cells inside the first knot, the numerical emissivity is of order $10^{-13} \mathrm{erg} \mathrm{cm}^{-2} \mathrm{~s}^{-1}$, which is in good agreement with data from HH34, see Morse et al., 1992.

In order to obtain a correct spatial spacing of our knots, while keeping unchanged the other parameters, we had to reduce the size of the nozzle to $2 \cdot 10^{14} \mathrm{~cm}$. This case is shown in Fig. 4 to 6 .

Fig. 4 displays the density map, after $\approx 700$ years, showing a well formed pattern of internal oblique shocks. It is worth noticing that, though $r_{j e t}$ is much smaller, the effective beam radius is $\approx 2 \cdot 10^{15} \mathrm{~cm}$.

Fig. 5 shows the total density, the electron density and the temperature measured along the axis of the jet. The adiabatic expansion, which is due to the strong pressure ratio, and causes all the values to drop, is clearly visible. The electron density, in particular, falls to zero, so that all the electrons in the emitting regions are due to post-shock ionization, independently of the inflow ionization fraction.

Four knots are clearly visible. The spacing is $\approx 5 "$ and the emissivity, shown by Fig. 6, is again significant and in the range of observed values.

\section{Conclusions}

Our numerical simulations of radiative, non-equilibrium jets show that the internal oblique shocks survive the cooling losses and, with the proper choice of the inflow parameters, are responsible for the formation of a pattern of optical knots whose intensity and spacing are in good agreement with observations. We conclude that they could explain the internal knotty emissivity often observed in many $\mathrm{HH}$ jets, even if other effects, namely the magnetic field, $3 D$ effects and low-frequency fluctuations of the inflow conditions, should be invoked to give a more general description of these objects.

Acknowledgements: The authors wish to thank Francesca Bacciotti, Tino Oliva and Claudio Chiuderi for their useful and friendly suggestions. 


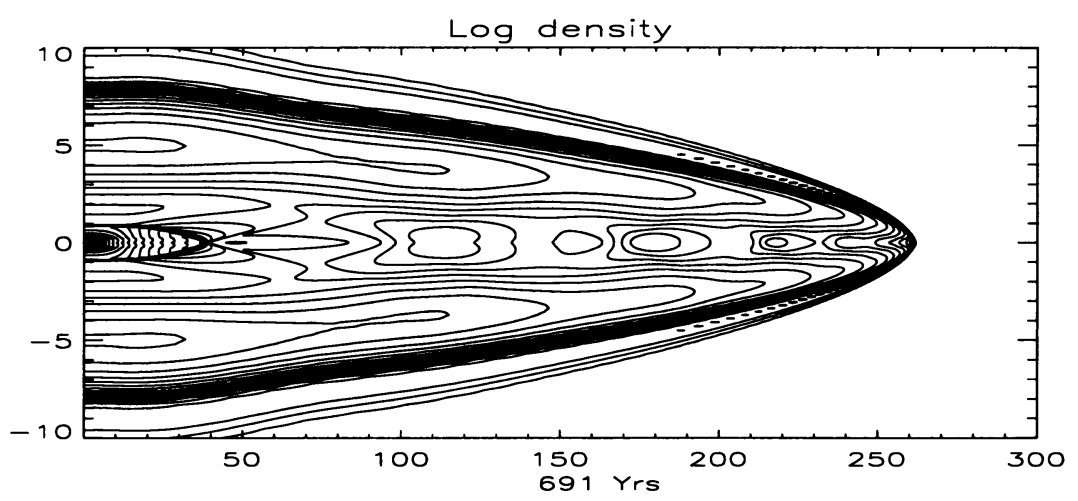

Figure 4. Density map (logarithmical) for the strongly under-expanded case with nozzle radius $r_{j e t}=0.210^{15} \mathrm{~cm}$

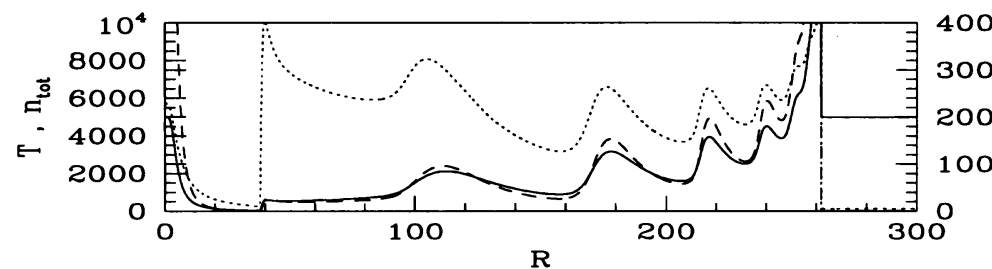

Figure 5. Profile along the jet axis of temperature (dotted line), electrons density (in $\mathrm{cm}^{-3}$, dashed line) and total density (in $\mathrm{cm}^{-3}$, full line).

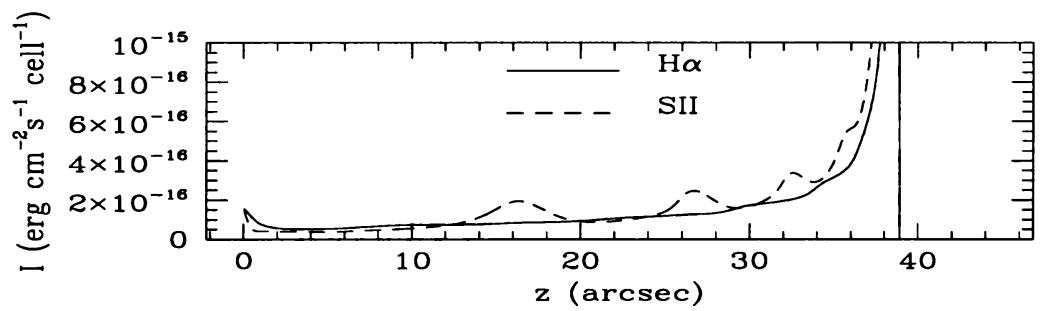

Figure 6. Profile along the jet axis of the [SII] (dashed line) and $\mathrm{H}_{\alpha}$ (full line) fluxes, at a distance of $460 \mathrm{pc}$, from a cylindrically symmetric slice normal to the axis, with a width of 1 cell $\left(1.2 \times 10^{14} \mathrm{~cm}\right)$. 


\section{References}

Bacciotti F., Chiuderi C., Oliva E., 1995, A\& A 296, 185

Bacciotti F., Eislöffel J., 1997, A\& A, submitted.

Blondin J.M., Fryxell B.A., Königl A., 1990,ApJ 360, 370

Cantó J., Raga A.C., Binette,L. 1989, Rev. Mex. Ast. 17, 65

Dalgarno A., Mc Cray R.A., 1972, ARAA 10, 375

Einfeldt B., Muntz C.D., Roe P.L., Sjogreen B., 1991, Journal of Computational Physics 92, 273

Hardee P., Stone J., Rosen A., 1997, in 'Low Mass Star Formation from Infall to Outflow', Poster Proceedings IAU Symposium 182, ed. Malbet F., Castets A., Grenoble.

Heathcote,S. et al.,1996, AJ 112,1141

Hollenbach D., McKee C. F., 1989, ApJ 299, 306

Lepp S., Shull J.M., 1983, ApJ 270, 578

Lorusso, S., 1996, Tesi di laurea, Univ. Firenze

Mac Low M., Shull J.M., 1986, ApJ 302, 585

Martin P.G., Schwarz D.H., Mandy M.E., 1996, ApJ 461, 265

Morse J.A., Hartigan P., Cecil G., Raymond J.C., Heathcote S., 1992, ApJ 399, 231

Reipurth B., Heathcote S., 1992, A\& A 257, 693

Sofia U.J., Cardelli J.A., Savage B.D., 1994, ApJ 430, 650

Shu C.W., Osher S., 1988, Journal of Computational Physics 77, 439

Stone J.M., Norman M., 1993, ApJ 413, 210

Suttner G., Smith D.M., Yorke H.,W., 1997, in 'Low Mass Star Formation from Infall to Outflow', Poster Proceedings IAU Symposium 182, ed. Malbet F., Castets A., Grenoble

Van Leer B., 1979, Journal of Computational Physics 32, 101 